\title{
Phase I/II Trial
}

National Cancer Institute

\section{Source}

National Cancer Institute. Phase I/II Trial. NCI Thesaurus. Code C15693.

A clinical research protocol designed to study the safety, dosage levels and response to new treatment. Phase I/II trials combine a Phase I and a Phase II trial of the same treatment into a single protocol. 